https://doi.org/10.22319/rmcp.v12i1.5088

Artículo

\title{
Las funciones de las aves en la producción avícola de pequeña escala: el caso de una comunidad rural en Hidalgo, México
}

\begin{abstract}
Ana Rosa Romero-López ${ }^{a^{*}}$
${ }^{\text {a } U n i v e r s i d a d ~ N a c i o n a l ~ A u t o ́ n o m a ~ d e ~ M e ́ x i c o . ~ F a c u l t a d ~ d e ~ M e d i c i n a ~ V e t e r i n a r i a ~ y ~ Z o o t e c n i a . ~}$ Ciudad de México, México.
\end{abstract}

*Autor de correspondencia: anarosa.romero.lopez@gmail.com

\section{Resumen:}

En México, diversos estudios enfatizan que la importancia de la avicultura de pequeña escala en el ámbito rural recae en dos funciones que se les asignan a las aves: 1) aporte a la seguridad alimentaria y 2) generación de ingresos económicos. Pocos son los estudios que identifican y describen las funciones complementarias que tienen las aves en sistemas de producción de pequeña escala y la manera en que dicha especie contribuye al bienestar de la familia productora. En el presente estudio se identificaron y analizaron las funciones que las familias productoras le asignaban a la especie avícola en las 26 unidades de producción que poseían aves, de un total de 29, en la comunidad rural La Cuchilla, Hidalgo, México. Se encontró que las aves podían tener 6 diferentes funciones, no mutuamente excluyentes: nutrimental (26.9\%), medioambiental (24.4\%), cultural (17.9\%), económica (16.7\%), social (11.5\%) y de recreación (2.6\%). Cada una de las funciones contribuían al bienestar familiar en cinco diferentes esferas: seguridad alimentaria, disponibilidad de recursos económicos, reforzamiento de relaciones sociales, vinculación al mercado y transmisión de conocimientos vernáculos. Se concluye que las aves pueden tener una multifuncionalidad que va más allá de su aporte a la seguridad alimentaria (función nutrimental) y generación de recursos económicos (función económica). Las funciones de las aves pueden ser estrategias que los productores definen para alcanzar el bienestar familiar en cinco diferentes esferas. La comprensión integral del concepto de función es importante para el diseño de estrategias de desarrollo acordes a los objetivos y lógica de producción de la familia productora.

Palabras clave: Traspatio, Desarrollo rural, Seguridad alimentaria, Avicultura familiar. 
Recibido: 30/09/2018

Aceptado: 07/11/2019

\section{Introducción}

La avicultura de pequeña escala (APE) es una de las actividades pecuarias con mayor presencia en el sector rural, una de sus particularidades radica en que sus formas de producción responden a las necesidades de la familia productora y no tanto a la satisfacción de las demandas del mercado. Diversos autores resaltan dos funciones primordiales que tiene la producción avícola en comunidades rurales. Por un lado, su aporte de proteína de alta calidad y de bajo costo a la dieta familiar y por otro, los ingresos extras generados por la venta de sus productos, siendo estos últimos los que permiten la adquisición de bienes y servicios complementarios que satisfacen las necesidades familiares ${ }^{(1,2)}$.

Centeno $^{(3)}$ propone que los animales domésticos en un sistema de producción de pequeña escala pueden satisfacer funciones más allá de las nutricionales (consumo familiar) y de las económicas (venta para obtención de dinero o trueque), tales como son las socioculturales (uso en festividades comunitarias, celebraciones familiares, instrumento de educación). Por su parte otros autores proponen diversas funciones que también pueden tener los animales domésticos, que contribuyen al bienestar familiar y que responden a las razones por las cuales las familias productoras tienen animales en su unidad de producción, tales como son: función medioambiental (obtención de estiércol), de trabajo (carga, tracción, entre otros), de ahorro, de seguro (al proveer a la familia de bienes en tiempos de crisis) y social (reforzamiento de lazos y jerarquía social $)^{(4,5)}$.

Las funciones que la familia productora le asigna a los animales domésticos pueden ser diversas y estar influenciadas por sus estrategias, las cuales se establecen para alcanzar un beneficio específico que contribuye al bienestar familiar. Así, cada estrategia estará determinada por las capacidades de la familia productora (disponibilidad de mano de obra, experiencia, conocimientos), sus necesidades (ingreso económico, servicios de salud, obtención de alimento, entre otros), recursos, objetivos particulares y valores ${ }^{(3,5)}$.

En el caso de las aves, si bien la función y la dinámica de su producción a pequeña escala está estrechamente ligada a las características y necesidades de sustento y bienestar de la familia productora, son pocos los estudios en México que identifican y analizan las funciones

que a las aves se les asigna, más allá del discurso redundante de su función nutrimental y económica, y la manera en que la que dicha producción favorece el bienestar familiar. 
El presente estudio pretende identificar las funciones que se le asignan a las aves, en la comunidad rural La Cuchilla, Nopala de Villagrán, Hidalgo, México a partir de las funciones que proponen diversos autores ${ }^{(4,6)}$ y la manera en que éstas contribuyen al bienestar de las familias que desarrollan la avicultura.

\section{Material y métodos}

\section{Área de estudio}

El presente trabajo se llevó a cabo en la comunidad rural La Cuchilla, considerada como de alta marginación, en el municipio de Nopala de Villagrán, Hidalgo. La Cuchilla es una de las 112 comunidades pertenecientes al municipio de Nopala de Villagrán que, de acuerdo con INEGI, corresponde al segundo municipio con mayor número de aves de corral del estado de Hidalgo (689,850 cabezas) al concentrar el $18 \%$ del total de aves del estado. Dicha comunidad cuenta con un clima templado subhúmedo con lluvias en verano y está catalogada como una comunidad rural con grado de marginación alto ${ }^{(7)}$.

\section{Etapas de investigación y recolección de datos}

El estudio se desarrolló bajo un diseño no experimental usando un método descriptivo. En la primera etapa se realizó un censo agropecuario para la identificación del número total de unidades de producción familiar (UPF) y tipo y número de especies animales presentes en la comunidad. Posteriormente se seleccionaron las UPF que llevaban a cabo la producción avícola (26/29), con el fin de identificar la presencia de aves.

En la segunda etapa se aplicaron cuestionarios semiestructurados a la persona responsable de la producción avícola de las unidades de producción seleccionadas, con el fin de identificar las funciones que se les asignaba a las aves, siguiendo la clasificación propuesta por diversos autores $^{(4,6)}$. Las funciones de las aves se definieron como aquel rol que la familia les asignaba a sus aves según los beneficios que esperaban obtener de su cría y producción. Para identificar y clasificar las funciones que se les asignaba a las aves, se definieron preguntas semiestructuradas que dieran información sobre los siguientes aspectos.

Función nutrimental: destino de la producción para consumo familiar, cantidad de la producción destinada al consumo familiar y frecuencia de consumo de los productos avícolas.

Función cultural: grado de involucramiento de los hijos a la actividad avícola y razones para integrar a los hijos a las actividades de cría y producción avícola.

Función económica: porcentaje de la producción destinada a la venta. 
Función social: destino de la producción para celebraciones, fiestas (boda, bautismo, cumpleaños) o regalo a vecinos o familiares.

Función medioambiental: destino de la gallinaza o pollinaza.

Función de recreación: cría de aves por placer.

Las esferas del bienestar familiar se definieron según lo reportado en estudios que denotan los aportes de la ganadería de pequeña escala a nivel familiar: seguridad alimentaria ${ }^{(8,9)}$, disponibilidad de recursos económicos ${ }^{(10)}$, reforzamiento de relaciones sociales ${ }^{(11)}$, vinculación al mercado ${ }^{(8)}$ y transmisión de conocimientos vernáculos ${ }^{(5)}$. Las esferas del bienestar familiar se consideraron como aquellos impactos positivos que conllevaba el cumplimiento de las funciones de las aves en la vida cotidiana de la familia productora.

Las estrategias utilizadas para la venta del huevo de gallina se identificaron mediante preguntas semiestructuradas con respecto a los puntos de venta (directos e indirectos), los tipos de consumidores y la periodicidad de venta. Dichas estrategias de venta se clasificaron según lo propuesto por $\operatorname{LEADER}^{(12)}$.

\section{Manejo de datos y análisis}

Los datos recolectados se analizaron a partir de estadística descriptiva (frecuencias absolutas y relativas) a través del programa estadístico para las ciencias sociales SPSS 15.0 y Excel 2010.

\section{Resultados}

\section{Características generales de las unidades de producción familiar (UPF)}

El $93.1 \%(27 / 29)$ de las UPF reportaron tener presencia de animales. El total de animales existentes en la comunidad fueron 1,089 y la especie animal con mayor número de cabezas fueron las aves (48\%), seguidas de ovinos (39\%), bovinos productores de leche $(11 \%)$, conejos $(1 \%)$, caballos $(0.6 \%)$ y cerdos $(0.3 \%)$. Del total de las UPF que contaban con animales, el $96.3 \%(26 / 27)$ poseían aves, de las cuales el $96.15 \%$ se dedicaban a la producción de huevo y $3.85 \%$, carne. La especie avícola más abundante fueron las gallinas para la producción de huevo $(71 \%)$, seguida de pollos $(10.4 \%)$, gallos $(6.4 \%)$, guajolotes $(6.1 \%)$, aves de pelea $(4.7 \%)$, patos $(0.8 \%)$ y gansos $(0.6 \%)$. 


\section{Miembros de la familia involucradas en la UPF}

En el $100 \%$ de las UPF la actividad avícola era responsabilidad de las mujeres. Sin embargo, en algunos casos la mujer entrevistada se encargaba en su totalidad de las aves (57\%) y en otros, la mamá (4\%), el esposo (14\%), los hijos pequeños (14\%) e hijas adultas (11\%) se involucraban en la producción avícola y apoyaban en la realización de determinadas actividades como: recolección de huevo, limpieza de corrales, alimentación, limpieza de bebederos y comederos y venta de huevo, ya fuera de manera constante o esporádica.

\section{Importancia de las aves}

Las UPF con presencia de animales contaban con $2.4 \pm 0.9$ especies animales en un rango de entre 1 a 6 especies, por lo que la actividad pecuaria en la comunidad tendía más a la diversificación que a la especialización de su producción. En general, la especie animal considerada como la más importante fueron las aves (Cuadro 1), sin embargo, el porcentaje de UPF que consideraba a las aves como las más importantes, era mayor en aquellas con presencia de menos de tres especies animales $(82.3 \%)$. Lo anterior muestra que conforme la diversidad de especies animales incrementaba (entre 4 a 6), la importancia que se le atribuían a las aves disminuía (17.7\%).

Cuadro 1: Actividad pecuaria que se considera como la más importante para las productoras entrevistadas de la comunidad La Cuchilla

\begin{tabular}{lcc}
\hline \multicolumn{1}{c}{ Actividad pecuaria } & Frecuencia & \% \\
\hline Aves & 17 & 63.0 \\
Bovinos productores de leche & 7 & 25.9 \\
Ovinos & 2 & 7.4 \\
Conejos & 1 & 3.7 \\
Total & 27 & 100.0 \\
\hline
\end{tabular}

Las razones por las cuales el $63 \%$ de las productoras consideraban a la avicultura como la actividad más importante en comparación con las demás actividades en sus UPF, era debido a los diversos beneficios que éstas obtenían de dicha actividad, resaltando el aporte a la seguridad alimentaria como el beneficio más mencionado (Cuadro 2). 
Cuadro 2: Razones por las cuales las aves son consideradas las más importantes para las productoras entrevistadas en la comunidad La Cuchilla

\begin{tabular}{lcc}
\hline \multicolumn{1}{c}{ Razones } & Frecuencia & $\%$ \\
\hline $\begin{array}{l}\text { Es una fuente de alimento para la familia (aporte a la seguridad } \\
\text { alimentaria) }\end{array}$ & 9 & 37.4 \\
$\begin{array}{l}\text { Las gallinas representan un alimento ante la escasez de comida } \\
\text { La venta de huevo y pollitos permite la obtención de dinero }\end{array}$ & 1 & 4.2 \\
para la satisfacción de necesidades familiares & 4 & 16.6 \\
$\begin{array}{l}\text { Evita la compra externa de pollo y huevo } \\
\text { Posibilidad de venta de un ave o borrego en emergencias } \\
\text { familiares }\end{array}$ & 1 & 4.2 \\
$\begin{array}{l}\text { Es un alimento para ocasiones especiales (visita de hijos, } \\
\text { celebraciones, fiestas familiares) }\end{array}$ & 1 & 4.2 \\
$\begin{array}{l}\text { Se entretiene y es un pasatiempo } \\
\text { Es su única especie }\end{array}$ & 1 & 4.2 \\
$\begin{array}{l}\text { Proporciona un sentido de propiedad } \\
\text { Permite la obtención de huevo }\end{array}$ & 2 & 4.2 \\
Son consideradas parte de la familia & 2 & 8.3 \\
TOTAL & 1 & 4.2 \\
\hline
\end{tabular}

\section{Las funciones de las aves en el sistema de producción de pequeña escala}

De acuerdo a los diversos usos y destinos que las productoras le daban a los productos avícolas y a las aves, las funciones de la especie avícola se clasificaron en seis (Cuadro 3). En promedio cada productora les asignaba tres funciones a las aves, siendo éstas no mutuamente excluyentes. 
Cuadro 3: Las funciones que se les asignan a las aves las productoras entrevistadas de la comunidad La Cuchilla

\begin{tabular}{llcc}
\hline & Función & Frecuencia & \% \\
\hline Nutrimental & $\begin{array}{l}\text { Consumo de productos } \\
\text { avícolas a nivel familiar }\end{array}$ & 21 & 26.9 \\
& $\begin{array}{l}\text { Uso del excremento como } \\
\text { abono natural para su uso }\end{array}$ & 19 & 24.4 \\
& en la milpa \\
& Transmisión de \\
conocimiento vernáculo, & & \\
enseñanza de ética de \\
trabajo y valores a través \\
de la cría y cuidado de las \\
aves \\
Venta de productos \\
avícolas para la obtención \\
de ingresos económicos \\
Uso de los productos \\
avícolas en celebraciones \\
(boda, fiestas,
\end{tabular}

a) Función nutrimental

El consumo familiar de carne de ave proveniente de su propia producción fue en promedio 1 vez cada $24 \pm 5.61$ semanas ( 6 meses). El bajo consumo de carne al mes era debido a que su consumo se realizaba únicamente en situaciones específicas como lo era en época de escasez de alimentos o por un simple gusto, principalmente (Cuadro 4). Además, la función prioritaria de las aves no era la de proveer carne para el consumo familiar sino más bien era la producción de huevo. 
Cuadro 4: Razones de consumo de carne de gallina proveniente de la unidad de producción La Cuchilla

\begin{tabular}{lcl}
\hline \multicolumn{1}{c}{ Razones } & Frecuencia & \% \\
\hline Escasez de comida & 7 & 26.92 \\
Gusto & 7 & 26.92 \\
Aves viejas, que ya no ponen o están enfermas & 5 & 19.23 \\
Celebración (cumpleaños, visita de algún familiar) & 4 & 15.38 \\
Cuando no hay dinero para comprar carne & 1 & 3.85 \\
No consume & 1 & 3.85 \\
Evitar la compra de carne "de granja” y evitar el gasto & 1 & 3.85 \\
Total & 26 & 100.0 \\
\hline
\end{tabular}

En este sentido, el $96.15 \%$ de las UPF con aves se dedicaban a la producción de huevo cuyos diferentes usos y destinos eran principalmente para consumo familiar (54.76\%), para venta (35.71\%) y como regalo a sus vecinos o para sus familiares que ya no habitaban en la comunidad, y con los cuales se buscaba reforzar lazos de reciprocidad, confianza y trato entre ellos $(9.53 \%)$.

b) Función cultural

El $100 \%$ de las productoras que tenían algún miembro de la familia menor a 17 años, ya fuera de la familia nuclear o extensa, utilizaban a la avicultura como medio para transmitir valores y enseñar ética de trabajo a partir del involucramiento de sus integrantes de la familia en el cuidado y producción de las aves. Dentro de los elementos trasmitidos se mencionaron: 1) Enseñar a trabajar a los hijos desde temprana edad y a hacerlos pensar en que deben realizar una actividad en la casa para que ocupen su mente en algo positivo que les ayude en un futuro; 2) Enseñar que es un ahorro; 3) Preservar de generación en generación la cultura y el conocimiento de que a partir de trabajo y esfuerzo, los hijos pueden obtener su propio alimento; 4) Enseñar a cuidar a los animales para poder obtener beneficios tangibles (dinero, alimentos de origen animal) o intangibles (conocimiento, experiencia en el cuidado de los animales); 5) Enseñar el trabajo que cuesta tener las cosas y a saberlas valorar; 6) Dejar un legado para los hijos, que pueda ser trabajado continuamente para que constituya un medio de vida para el futuro.

c) Función económica

Del total de UPF que llevaban a cabo la producción de huevo, el $60 \%$ destinaban parte de su producción para la venta a nivel local. El precio de venta de huevo era de $\$ 2.00 /$ pieza en toda la comunidad, a excepción de una productora, quien mencionó venderlo a \$2.50/pieza. Los ingresos económicos generados a partir de la venta de huevo oscilaban entre \$20 hasta \$420 pesos por semana. La variación existente entre ingresos económicos obtenidos era debido a que estos dependían de la cantidad de gallinas presentes por UPF y el porcentaje de huevo destinado a la venta. 
Para la venta de huevo, las pequeñas productoras utilizaban diferentes circuitos cortos de comercialización (CCC) que las enlazaba con consumidores dentro y fuera de la comunidad rural. Se identificaron $10 \mathrm{CCC}$, los cuales se clasificaron en tres grupos, de acuerdo a LEADER $^{(12)}$ : venta a mercados locales $(25.92 \%)$, venta directa en la UPF $(55.56 \%)$ y entrega a domicilio (18.52\%) (Cuadro 5).

Cuadro 5: Circuitos cortos de comercialización (CCC) de huevo utilizados por productoras de la comunidad La Cuchilla

\begin{tabular}{llcl}
\hline \multicolumn{1}{c}{ CCC } & \multicolumn{1}{c}{ Lugar/Actor } & Frecuencia & \% \\
\hline $\begin{array}{l}\text { Venta en } \\
\text { tiendas }\end{array}$ & Tienda de abarrotes y carnicería & 2 & 7.41 \\
& Tianguis & 3 & 11.11 \\
CCC1 & Restaurantes & 1 & 3.70 \\
$(25.92 \%)$ & Pastelería & 1 & 3.70 \\
Venta directa & Vecinos para su autoconsumo & 8 & 29.63 \\
en la UPF & Miembros de la familia de otro estado o municipio & 4 & 14.81 \\
CCC2 & Vecinos para completar sus pedidos (cooperación) & 2 & 7.41 \\
$(55.56 \%)$ & Visita en la UPF & 1 & 3.70 \\
$\begin{array}{llll}\text { Reparto a } \\
\text { domicilio }\end{array}$ & Personas específicas por pedidos previos & 3 & 11.11 \\
CCC3 & Casa por casa en el municipio & 2 & 7.41 \\
$(18.52 \%)$ & & & \\
\hline
\end{tabular}

Si bien el $60 \%$ de las UPF vendían su huevo, no toda la producción se destinaba para su venta, sino que el número de huevos obtenidos se distribuía dependiendo del beneficio que cada productora pretendía obtener. De acuerdo al número de huevos que destinaba las productoras para su venta fue posible dividir las UPF en tres grupos:

Grupo 1 (Económica): productoras que destinaban el mayor número de huevos para su venta a nivel local (66.67 \%) (Cuadro 6). 
Cuadro 6: Circuitos cortos de comercialización (CCC) utilizados por las productoras entrevistadas que vendían huevo y cuyo mayor número de huevos producidos eran destinados para la venta

\begin{tabular}{llll}
\hline Tipo de función & \multicolumn{1}{c}{ CCC } & \multicolumn{1}{c}{ Lugar/Actor } & \% \\
\hline & CCC2 & Vecinos & 28.57 \\
& & Cooperación & 9.52 \\
& $52.38 \%$ & Familiares & 9.52 \\
& & Visita a UPF & 4.76 \\
Grupo 1. & $\mathrm{CCC} 1$ & Pastelería & 4.76 \\
Económica & $28.57 \%$ & Tianguis & 14.29 \\
& & Tienda de abarrotes & 9.52 \\
& \multirow{2}{*}{$\mathrm{CCC} 3$} & Pedidos previos & 14.29 \\
& \multirow{2}{*}{$19.05 \%$} & Venta casa por casa & 4.76 \\
\hline
\end{tabular}

Grupo 2 (Consumo familiar): productoras que destinaban el mayor número de huevos para el consumo familiar $(26.67 \%)$ (Cuadro 7$)$.

Cuadro 7: Circuitos cortos de comercialización (CCC) utilizados por las productoras entrevistadas que vendían huevo, pero cuyo mayor número de huevos producidos eran destinados para consumo

\begin{tabular}{llll}
\hline \multicolumn{1}{c}{ Tipo de función } & \multicolumn{1}{c}{ CCC } & \multicolumn{1}{c}{ Lugar/Actor } & $\%$ \\
\hline \multirow{3}{*}{ Grupo 2. } & CCC2 & Familiares & 40 \\
& $60 \%$ & Vecinos & 20 \\
Consumo familiar & $\mathrm{CCC} 1$ & Restaurante & 20 \\
& $20 \%$ & & \\
& $\mathrm{CCC} 3$ & Venta casa por casa & 20 \\
& $20 \%$ & & \\
\hline
\end{tabular}

Grupo 3 (Social); productoras que destinaban el mayor número de huevos para regalo a familiares o vecinos cercanos $(6.67 \%)$.

Como lo muestran el Cuadro 6 y 7, la diversidad de lugares utilizados para la venta del huevo difería según el grupo al que cada UPF pertenecía. Por ejemplo, el grupo 1 tenía una mayor 
diversidad de lugares para la venta de huevo por CCC en comparación con el grupo 2. El grupo 3 lo conformaba solo una UPF que destinaba el mayor número de huevos para regalarlo a sus familiares. Lo anterior indica que a pesar de que existían UPF que vendían el huevo, no todas ellas destinaban la mayor parte de su producción para la venta por lo que privilegiaban otro tipo de funciones tales como son la social o nutrimental.

d) Función social

Como se mencionó, un porcentaje del huevo producido en las UPF se le asignaba una función social debido a que este era consumido en eventos familiares (visitas de parientes cercanos o cumpleaños), así como también se destinaba para regalo a familiares o vecinos con el objetivo de reforzar los lazos de reciprocidad, confianza y trato entre ellos. En este caso, no se reportó el consumo de carne de ave en eventos especiales.

e) Función medioambiental

El $83.3 \%$ de las productoras utilizaba la gallinaza como abono para su milpa o para la milpa de un familiar, el $8.3 \%$ no le daba ningún uso, el $4.2 \%$ la vendía o usaba en el herbario (4.2\%). Por otra parte, el $52 \%$ de los productores utilizaban el maíz que ellos mismos producían en su milpa para alimentar a las aves, lo que permitía disminuir la compra de insumos externos. Cabe mencionar que dicha relación se veía afectada en determinadas épocas del año (temporadas de no siembra o escasez de agua).

f) Función de recreación

De acuerdo con $2.6 \%$ de las entrevistadas, la avicultura era una actividad de recreación y relajante, que les permitía descansar de sus actividades cotidianas y la cual les generaba gusto y satisfacción.

\section{Esferas del bienestar familiar}

Cada una de las funciones de las aves contribuían al bienestar familiar en diferentes vertientes, las cuales se clasificaron en cinco esferas: seguridad alimentaria, disponibilidad de recursos económicos, reforzamiento de relaciones sociales, vinculación al mercado y transmisión de conocimientos vernáculos.

Seguridad alimentaria (disponibilidad y accesibilidad): el huevo o la carne de las aves se consumía en época de escasez de comida, por gusto, como ahorro o cuando no había disponibilidad de dinero.

Disponibilidad de recursos económicos: los ingresos económicos obtenidos por cada UPF eran utilizados para obtener diversos bienes y servicios que permitían mejorar el bienestar familiar, también favorecían la reinversión en las diversas actividades pecuarias de la familia 
y contribuían a la seguridad alimentaria al permitir la compra de alimentos que no eran producidos dentro de la UPF (Cuadro 8).

Cuadro 8: Destino de los ingresos económicos obtenidos por las productoras entrevistadas a partir de la venta de huevo en la comunidad La Cuchilla

\begin{tabular}{lll}
\hline Uso de los recursos económicos & Frecuencia & \% \\
\hline Compra de alimento para la familia & 12 & 57.14 \\
Compra de alimento para aves & 4 & 19.06 \\
Compra de alimento para otras especies & 1 & 4.76 \\
Pasaje & 2 & 9.52 \\
Pasaje de hijos para la escuela & 1 & 4.76 \\
Pago del molino & 1 & 4.76 \\
Total & 21 & 100 \\
\hline
\end{tabular}

Reforzamiento de relaciones sociales: el huevo producido se utilizaba como regalo a los vecinos para reforzar lazos de reciprocidad, confianza y trato entre ellos o para sus familiares que ya no habitaban en la comunidad.

En este caso, fue posible inferir que la producción de huevo permitía el establecimiento de relaciones estrechas entre productoras a partir de mecanismos de cooperación para la venta de huevo, en donde una productora podía completar el número de piezas de huevo a otra productora para que esta pudiera vender la cantidad de huevos que requería (estrategias de cooperación).

Vinculación al mercado: Las pequeñas productoras utilizaban diferentes CCC para la venta de su huevo. El tipo de circuitos utilizados por las productoras para comercializar su huevo, incluían estrategias que las conectaban tanto con consumidores dentro de la comunidad como fuera de esta. De acuerdo con sus criterios, las ventajas de los circuitos comerciales empleados para la venta de huevo eran la inexistencia o escaso número de intermediarios involucrados para la comercialización del huevo lo que permitía vender de manera directa el huevo a los consumidores y concentrar de las ganancias obtenidas por parte del productor. Transmisión de conocimientos vernáculos: las productoras que tenían algún miembro de la familia menor a 17 años, ya fuera parte de la familia nuclear o extensa, utilizaban a la avicultura como medio para transmitir valores y enseñar ética de trabajo a partir del involucramiento de sus integrantes de la familia en el cuidado y producción de las aves, así como también para heredar conocimientos vernáculos sobre formas de producción y medios de subsistencia. 


\section{Discusión}

\section{Características generales de las UPF de la comunidad rural}

Los datos disponibles y generados a nivel nacional que denotan la situación de las comunidades rurales en México corresponden únicamente a la densidad poblacional, grado de marginación, grado de rezago social, número de viviendas e indicadores de carencia de vivencia. Dado lo anterior, hay una escasez en la disponibilidad de datos sobre la producción y productividad de las actividades agropecuarias y no agropecuarias de las comunidades rurales, pues dichos datos se conocen por municipio o por estado, pero no por comunidad rural.

La búsqueda de datos a nivel micro sobre la producción avícola en una comunidad rural se dificulta, pues la información que proveen los censos de INEGI tiene las limitantes que a continuación se describen: 1) La información que se provee es únicamente a nivel municipal y se centra en la densidad poblacional de sus aves, cantidad de venta de los productos avícolas y su acceso a tecnología. 2) Los datos corresponden únicamente a aquellas unidades de producción que reportan la existencia de más de 1,000 aves, situación que ignora a aquellas producciones de pequeña escala que poseen un número pequeño de aves. 3) Los datos no permiten distinguir la dinámica entre sistemas de producción avícola a gran escala o de pequeña escala, según su acceso a tecnologías o densidad animal

En el caso específico de la comunidad La Cuchilla, no hay datos previamente existentes que ayuden a vislumbrar las características generales de producción de sus actividades agropecuarias y no agropecuarias ni de su nivel tecnológico, su productividad o la generación de ingresos o de empleos.

Antes este escenario, es posible determinar la importancia de trabajos de investigación, como el presente estudio, que se adentren a la recolección y análisis a nivel micro de las comunidades rurales y que proporcionen información básica y fundamental para el diseño de estudios más complejos que ayuden a entender la dinámica de las UPF de comunidades rurales a nivel local, nacional e internacional y la forma en la que perviven en un sistema económico capitalista.

Los datos que en el presente estudio se reportan sobre la comunidad rural La Cuchilla, permiten vislumbrar las actividades de la comunidad y la importancia de la producción de aves para las familias productoras. 


\section{Importancia de las aves}

Diversos estudios realizados en México resaltan dos razones comunes por las cuales la avicultura de pequeña escala existe en el sector rural, ya sea por consumo familiar y, en menor medida, por la venta de productos avícolas. Por ejemplo, en comunidades de Yucatán se reportó que el destino de los productos avícolas era para la venta, el consumo familiar y en algunos casos para incubación ${ }^{(13,14)}$.

En algunas comunidades de Oaxaca se ha resaltado que el destino de la producción de huevos ha sido para venta e incubación, mientras que en otras comunidades, por ejemplo Zompantle, la principal finalidad era la venta y la cría de polluelos ${ }^{(15)}$. En el caso de la costa del estado de Oaxaca se ha mencionado que la finalidad de las aves era la producción de alimentos para consumo familiar, venta u obsequio. En otras comunidades del mismo estado, se ha reportado que la producción ha sido esencialmente para consumo familiar y venta con los vecinos ${ }^{(16)}$. Los datos obtenidos en diferentes estudios realizados en México permiten inferir que las funciones que satisfacen las aves en el ámbito rural han sido reportadas únicamente por el destino que se les da a los productos avícolas y no han sido descritas, estudiadas o analizadas como un medio para establecer estrategias que permitan alcanzar el bienestar de las familias productoras.

En contraste a las contribuciones científicas que caracterizan de manera técnica a la avicultura de pequeña escala en México y que resaltan, principalmente, su aporte nutricional y económico a nivel familiar, diversos autores enfatizan que las aves deben ser consideradas como un medio para mejorar los modos de vida rurales y, por ende, el bienestar de las familias, pues las aves permiten el control de pestes, son proveedores de abono, son requeridas para festividades especiales y son esenciales para ceremonias tradicionales ${ }^{(6,11)}$.

Los resultados del presente estudio permiten vislumbrar que la familia productora, no necesariamente toma decisiones productivas guiadas por intereses de carácter técnicoeconómico, pues su fin principal no es alcanzar el máximo beneficio económico ${ }^{(17)}$, sino que también se toman en consideración cuestiones no económicas, como lo son las necesidades básicas de la persona y de su familia (nutricionales, económicas, sociales y culturales), para definir en mayor medida el carácter de su producción ${ }^{(18,19)}$ y las funciones que le asigna a los animales, de manera que los resultados del presente trabajo enfatizan la multifuncionalidad de las aves y no el fin zootécnico de las mismas.

a) Función nutrimental

Para mitigar el hambre y la malnutrición no solo es necesario producir más alimentos, sino también que estos satisfagan los gustos y preferencias de quien lo consume, además de hacerlos más disponibles para aquellos que lo necesitan ${ }^{(20)}$. Diversos autores concuerdan en 
que los sistemas de producción animal a pequeña escala tienen un potencial importante para contribuir a la seguridad alimentaria de los habitantes de países en vías de desarrollo ${ }^{(6,9,21,22)}$. Los datos del presente estudio coincidieron con dichos estudios en los que se indica que la avicultura ha contribuido a hacer más disponibles y accesibles la carne y huevo de gallina, particularmente cuando hay escasez de alimento.

\section{b) Función cultural}

La importancia que tienen las aves como medio para trasmitir una ética de trabajo o valores a los hijos o integrantes de la familia menores a 17 años no se encuentra como tal reportada en otros estudios, sin embargo, si existen estudios que consideran que el traspatio constituye un medio para la trasmisión de conocimientos vernáculos, valores y ética de trabajo a las generaciones de jóvenes para mantener vigente la cría de animales ${ }^{(5)}$.

Los datos que se muestran aquí permiten inferir la importancia de la avicultura como medio de educación informal a los jóvenes que pueden promover su arraigo al campo además de representar una opción de vida. Otros estudios resaltan el papel de las aves en la religión, en la medicina tradicional y como medios para consumir los desperdicios de las fincas ${ }^{(20)} \sin$ embargo en el presente estudio no se encontró ningún caso en el que se emplearan a las aves para dichos fines.

\section{c) Función económica}

La función económica de las aves se cumplía a través de la venta de huevo para la obtención de ingresos económicos inmediatos. En contraste con datos reportados en Oaxaca, donde el $50 \%$ de los huevos se destinaban a la venta y el otro $50 \%$ se destinaba a la incubación ${ }^{(15)}$, en el presente estudio el $60 \%$ de las productoras, destinaban parte de su producción para la venta de huevo a nivel local.

Similar a lo reportado en Etiopia ${ }^{(20)}$, el ingreso económico obtenido por la venta de los productos avícolas era utilizado principalmente para obtener bienes y servicios complementarios para cubrir las necesidades familiares como lo era la compra de alimentos para consumo familiar que no eran producidos en la UPF e inclusive para el pago de la molienda del maíz en el molino local ${ }^{(18,19)}$.

Algunos autores consideran que los pequeños productores están teniendo una reorientación de su perfil productivo hacia la generación de productos destinados al mercado debido a una mayor demanda de dinero en efectivo para hacer frente a nuevas necesidades y obligaciones que no estaban presentes en sociedades campesinas de principio de siglo ${ }^{(23)}$. Sin embargo, los datos del presente estudio mostraron que la función nutrimental no ha disminuido su importancia relativa en esta comunidad rural, más bien a partir de las diversas funciones que se les asignaban a las aves había sido posible la satisfacción de las necesidades de las productoras y sus familias sin ser la función económica la única vía para alcanzar el bienestar familiar. 
d) Función social

Los animales con funciones sociales se han utilizado principalmente para cumplir con las responsabilidades generadas por las relaciones sociales que establecen las familias con los demás miembros de la comunidad, como es el caso de las celebraciones familiares (bodas, bautizos, velorios), festividades locales (fiesta patronal o de barrio), tradiciones (día de muertos, semana santa), regalo o trueque ${ }^{(1,3,5)}$. Los datos del presente estudio mostraron que el consumo de huevo de ave se realizaba en eventos especiales (cumpleaños, festividades, entre otras) así como también se regalaban a familiares o vecinos con el fin de reforzar relaciones sociales entre las personas involucradas, datos que concordaron con estudios realizados en Asia y África ${ }^{(10,21,24)}$.

e) Función medioambiental

Las UPF estudiadas mostraron la posibilidad de llevar a cabo diferentes actividades tanto agropecuarias como no agropecuarias. La interrelación entre la ganadería y la agricultura era importante, ya que se reforzaban conexiones simbióticas entre ambas actividades agropecuarias. En el caso específico de las aves, según diversos estudios, su función medioambiental ha sido considerada importante por tres roles que estas pueden desempeñar: ser recolectoras de desperdicios de los granos y forrajes (evita presencia de fauna nociva) ${ }^{(25)}$, ser proveedoras de abono natural a la milpa y limpiadoras de malezas ${ }^{(20)}$. En este estudio, los resultados mostraron que el único rol importante de las aves dentro de su función medioambiental fue el uso que se le daba a las excretas como abono natural a la milpa lo que disminuía la generación de residuos de esta actividad.

f) Función de recreación

Algunos autores han reportado que la cría y producción de animales a pequeña escala permite la obtención de satisfactores tangibles e intangibles a las mujeres encargadas de dicha actividad. Dentro de los satisfactores intangibles se han reportado aquellos que proporciona bienestar a la mujer pero que no pueden ser transformados en dinero, sin embargo tienen un efecto directo en el estado anímico de las personas ${ }^{(26)}$. Otros estudios han identificado la adquisición de aves para fines de compañía o para combate, como en el caso de gallos de pelea, considerándolos como un medio de entretenimiento ${ }^{(27)}$. En el presente estudio, fue posible vislumbrar que específicamente la cría y producción de aves era considerado un medio de distracción para las mujeres productoras que generaba satisfacción y bienestar (beneficios intangibles). 


\section{Esferas del bienestar familiar}

Seguridad alimentaria: una familia tiene seguridad alimentaria cuando tiene acceso a los alimentos requeridos para una vida sana para todos sus miembros (adecuada en términos de calidad, cantidad, seguridad y culturalmente aceptados $)^{(9)}$. En este caso fue posible identificar que la producción avícola tiene un aporte a la seguridad alimentaria en las familias que llevan a cabo esta actividad, sin embargo, falta la evaluación de otros elementos que también forman parte del concepto de seguridad alimentaria, para determinar de manera más precisa su verdadero aporte.

Disponibilidad de recursos económicos: los productores en comunidades rurales frecuentemente no tienen acceso a mercados financieros, como los bancos. La ganadería ofrece una alternativa para ahorrar o acumular capital como una "fuente de ahorro vivo". La venta de especies como bovinos, cerdos y borregos permiten su transformación en dinero en tiempos de crisis. En este estudio, no se identificaron casos donde las aves se vendieran con el propósito de solventar una crisis financiera, pues las aves no poseen un valor económico alto por cabeza como los bovinos, sin embargo, sí fue posible identificar estrategias de venta de huevo para obtener dinero y con ello acceder a bienes y servicios complementarios ${ }^{(4)}$.

Reforzamiento de relaciones sociales: Los animales a los cuales se les asigna funciones sociales se utilizan principalmente para cumplir con las responsabilidades generadas por las relaciones sociales que establecen las familias con los demás miembros de la comunidad, como en celebraciones familiares (bodas, bautizos, velorios), festividades locales (fiesta patronal o de barrio), tradiciones (día de muertos, semana santa), regalo o trueque ${ }^{(1,3)}$. En este caso, fue posible inferir que la producción de huevo permitía el establecimiento de relaciones estrechas entre productoras a partir de mecanismos de cooperación para la venta de huevo.

Vinculación al mercado: La necesidad de obtener ingresos para responder a los cambios económicos, sociales, tecnológicos y políticos ha fomentado a que las pequeñas productoras desarrollen estrategias de comercialización para asegurar la generación de ingresos familiares y la continuidad tras generacional de la $\mathrm{UPF}^{(22)}$. Tal como lo muestran los datos del presente estudio, las productoras eran capaces de utilizar diversos canales cortos de comercialización para la venta de su producto avícola, lo que les permitió encontrar diferentes nichos de mercado que bien pueden permitir la venta de otros productos también generados en la UPF.

Transmisión de conocimientos vernáculos: En un estudio realizado en Yucatán, se señala que la pequeña escala tiene la función de dar cohesión a la unidad familiar y a la comunidad, a través de acciones para la preservación, enriquecimiento y difusión del saber de sus habitantes, ya que en el manejo de traspatio se refleja gran parte del conocimiento que poseen la familia tiene sobre su entorno ${ }^{(28)}$. En el presente estudio fue posible identificar los 
elementos esenciales que se trasmiten a través de la producción avícola como lo son valores, ética de trabajo y formas de producción.

Cabe mencionar que es necesario identificar los elementos particulares que se difunden, a través de esta producción pecuaria como lo son: estrategias de producción para disminuir la dependencia a insumos externos, tratamientos tradicionales a enfermedades, estrategias de venta etc.

Los esfuerzos para el desarrollo de la ganadería en países en vías de desarrollo han sido principalmente dirigidos a generar ingresos y satisfacer las necesidades crecientes de las unidades de producción. Estos esfuerzos generalmente dan prioridad a tecnologías que maximicen la productividad individual de los animales, a la transferencia de tecnología y con ello, a la difusión de información técnica ${ }^{(29,30)}$. Sin embargo, estas acciones no solo deben ir encaminadas al aumento del rendimiento de la producción avícola y aumentar la generación de ingresos, pues las UPF no necesariamente se desarrollan bajo una lógica capitalista y, por lo tanto, pueden no estar apropiadas al contexto en el que se desarrollan ${ }^{(4)}$. Las acciones deben ir acompañadas de una comprensión integral de la función del ave y el contexto en el que ésta se desarrolla para que el diseño e implementación de programas o estrategias den respuesta a problemas y preocupaciones de los productores y vayan acorde a sus objetivos y estrategias de producción ${ }^{(20,25)}$.

\section{Conclusiones e implicaciones}

Las productoras de la comunidad rural de La Cuchilla le podían asignar 6 diferentes funciones, no mutuamente excluyentes, a las aves: nutrimental, medioambiental, cultural, económica, social y de recreación. Cada una de las funciones contribuían al bienestar familiar en cinco diferentes esferas: seguridad alimentaria, disponibilidad de recursos económicos, reforzamiento de relaciones sociales, vinculación al mercado y transmisión de conocimientos vernáculos. Se concluye que las aves pueden tener una multifuncionalidad que va más allá de su aporte a la seguridad alimentaria (función nutrimental) y generación de recursos económicos (función económica). Las funciones de las aves pueden ser estrategias que los productores definen para alcanzar el bienestar familiar en cinco diferentes esferas. La comprensión integral del concepto de función es importante para el diseño de estrategias de desarrollo acordes a los objetivos y lógica de producción de la familia productora.

\section{Agradecimientos}

Al Dr Fernando Manzo Ramos por sus valiosos comentarios y recomendaciones en la propuesta y desarrollo de mi tesis de maestría "El sistema de producción avícola, las funciones de las aves, el mercado local y los circuitos cortos de comercialización; un estudio 
integral de la avicultura de pequeña escala en una comunidad rural de México", de la cual deriva la esencia de esta investigación.

\section{Literatura citada:}

1. Camacho M, Lezama P, Jeréz M, Kollas J, Vásquez M, García J, et al. Avicultura indígena mexicana: Sabiduría milenaria en extinción. Actas Iberoam Conserv Anim $2011 ; 375$.

2. Navarrete N, Sánchez E, Nava G, Espinoza A. Identificación y evaluación de indicadores en la crianza campesina. VII Congreso AMER; 2007.

3. Centeno S, Manzo F. Propuesta de modelo para el estudio de la ganadería en comunidades campesinas. Estudio de caso del ejido de Almeya, Puebla, México. En: Ganadería y seguridad alimentaria en tiempo de crisis. México: Universidad Autónoma de Chapingo; 2009.

4. Randolph T, Schelling E, Grace D, Nicholson C, Leroy J, Cole D, et al. Role of livestock in human nutrition and health for poverty reduction in developing countries. Am Soc Anim Sci. 2007;85:2788-2800.

5. García A, Guzmán E. La ganadería familiar, elemento cotidiano de los traspatios de la comunidad Juan Nepomuceno Álvarez, Copala, Guerrero, México. Cienc Biológicas 2014;1-11.

6. Mack S, Hoffman D, Otte J. The contribution of poultry to rural development. World Poult Sci Assoc 2005;61:7-13.

7. INEGI. Panorama agropecuario en Hidalgo. INEGI. México; 2012.

8. Cruz B, Muñóz M, Santoyo V, Martínez E, Aguilar N. Potencial y restricciones de la avicultura de traspatio sobre la seguridad alimentaria en Guerrero, México. Agric Soc Desarro 2016;13:257-275.

9. Bruyn J, Wong J, Bagnoi B, Pengelly B, Alders R. Family poultry and food security. CAB Reviews Perspectives in Agriculture Veterinary Science Nutrition and Natural Resources 2015;10(13):1-9.

10. Aklilu H, Udo H, Almekinders C, Van der Zipp A. How resource poor households value and access poultry: Village poultry keeping in Tigray, Ethiopia. Agric Syst 2008;96:175-183.

11. Alders R. Producción avícola por beneficio y por placer. Roma: FAO; 2005. 
12. LEADER. Comercialización de productos locales circuitos cortos y circuitos largos. Observatorio Europeo; 2000.

13. Gutiérrez M, Segura J, López L, Santos J, Santos R, Sarmiento L, et al. Características de la avicultura de traspatio en el municipio de Tetiz, Yucatán, México. Trop Subtrop Agroecosystems 2007;7:217-24.

14. Gutiérrez-Ruíz E, Aranda F, Rodríguez R, Bolio R, Ramírez S, Estrella J. Factores sociales de la crianza de animales de traspatio en Yucatán, México. Bioagrociencias 2012;5(1):20-28.

15. Chincoya H, Jeréz M, Herrera J, Mendoza P. Caracterización fenotípica y sistema de producción de las gallinas criollas en comunidades de Oaxaca. Rev Mex Agroecosistemas 2016;3(2):87-98.

16. Viveros J, Chávez J, Jeréz M, Villegas Y. Manejo de gallinas de traspatio en seis comunidades de los valles centrales de Oaxaca. Rev Mex Agroecosistemas. 2016;3(2):75-86.

17. López J, Damián M, Álvarez F, Parra F, Zuluaga G. La economía de traspatio como estrategia de supervivencia en San Nicolás de los Ranchos, Puebla, México. Rev Geogr Agríc 2012;48:51-62.

18. Shanin T. Naturaleza y lógica de la economía campesina. Argentina: Nueva Visión; 1976.

19. Chayanov A. Theory of Peasant Cooperatives. Great Britain: Ohio State University Press; 1991.

20. Tadelle D, Ogle B. Village poultry production systems in the central highlands of Ethiopia. Trop Anim Health Prod 2001;33:521-537.

21. Muchadeyi F, Sibanda S, Kusina J, Makuza S. The village chicken production system in Rushinga district of Zimbabwe. Livest Res Rural Develop 2004;16(6). Art. 40.

22. Mcainsh C, Kusina J, Madsen J, Nvoni O. Traditional chicken production in Zimbabwe. Worlds Poult Sci J 2004;60:233-246.

23. Cáceres D. Estrategias campesinas en sociedades rurales contemporáneas. Rev Fac Agron 15(1):67-72.

24. FAO. Family poultry development. Issues, opportunities and constraints. Animal Production and Health Working Paper. Animal Production and Health Working Paper; 2014. 
25. Moreki J, Petheram R, Tyler L. A study of small-scale poultry production systems in Serowe-Palapye sub-district of Botswanna. Livest Res Rural Develop 2010;22(3).

26. Vieyra J, Castillo A, Losada H, Cortés J, Alonso G, Ruíz T, et al. La participación de la mujer en la producción traspatio y sus beneficios tangibles e intangibles. Cuad Desarro Rural 2004;53:9-23.

27. Itza M, Carrera JM, Castillo Y, Ruíz O, Aguilar E, Sangines J. Caracterización de la avicultura de traspatio en una zona urbana de la frontera norte, México. Rev Científica 2016;26(5):300-305.

28. Molina M. Comparación de dos sistemas de producción y manejo sanitario de las aves criollas de traspatio en los municipios de Ignacio de la Llave y Teocelo, [tesis licenciatura].Veracruz: Universidad Veracruzana; 2013.

29. Elkashef O, Sarmiento L, Torres J. Backyard chicken production skills of rural women in Yucatán, México. Asian J Agric Ext 2016;10(1):1-12.

30. Salah O, Sarmiento L, Torres F, Fernandez L. Understanding the information acquisition sources of backyard chicken production practices amongst rural Mayan women in Yucatan, México. Agric Soc Desarro 2017;14:219-237. 